(PTG). We expect that a high rate of resilience is associated with

Phosphorus Absorption in Healthy Adults and in Patients with Moderate Chronic Kidney Disease

Elizabeth Stremke ${ }^{1}$, Gretchen Wiese ${ }^{1}$, Amy Wright ${ }^{1}$, Sharon Moe ${ }^{2}$, Ranjani Moorthi ${ }^{2}$ and Kathleen Hill Gallant ${ }^{1}$

${ }^{1}$ Purdue University and ${ }^{2}$ Indiana University School of Medicine

OBJECTIVES/SPECIFIC AIMS: The aim of this study is to compare intestinal phosphorus absorption in healthy adults and moderate stage chronic kidney disease patients in the context of a controlled feeding study METHODS/STUDY POPULATION: Participants are 30-75 years old and include 10 healthy subjects and 10 moderate-staged CKD patients. Each subject pool will be enrolled in a 9-day study period including 7 days of controlled feeding of a $1500 \mathrm{mg}$ phosphorus diet. Following the controlled feeding, two days of absorption tests will take place (oral and IV tests) utilizing radioisotopic phosphorus to calculate fractional absorption efficiency. RESULTS/ANTICIPATED RESULTS: Current enrollment has produced 7 total matched subject (current $n=14 / 20$ ). Four of the 7 pairs of completed subjects are female and 3 of 7 are black. Preliminary kinetic modeling data from the first enrolled subject show a moderate CKD patient with fractional absorption of 0.375 . With forthcoming analyses, we expect that this fractional absorption result will not be statistically different from this subject's matched pair, nor will each groups average absorption be different from the other. Additionally, we expect absorption to be maintained even with changes in secondary outcomes measures in serum (FGF23, 1,25-dihydroxyvitamin D, parathyroid hormone, and total phosphorus) in CKD patients. DISCUSSION/SIGNIFICANCE OF IMPACT: Lack of statistical difference in fractional phosphorus absorption between gropus would support that intestinal phosphorus absorption is inappropriately normal in CKD patients compared to healthy adults, despite evidence of abnormal phosphorus homeostatic mechanisms. Future studies will consider the effect of dietary $\mathrm{P}$ restriction, the most common nutrition intervention in moderate stage $\mathrm{CKD}$, on fractional absorption efficiency in CKD.

\section{Potentially traumatic events and its outcomes among help-seeking adults in Puerto Rico}

Marie Torres ${ }^{1}$, Alfonso Martinez-Taboas ${ }^{1}$, Coralee Perez-Pedrogo ${ }^{1}$ and Marisol Pena-Orellana ${ }^{1}$

${ }^{1}$ University of Puerto Rico-Medical Sciences Campus

OBJECTIVES/SPECIFIC AIMS: This study aims to evaluate potentially traumatic events (PTEs) and its relationship with posttraumatic stress symptoms (PTSS), posttraumatic growth (PTG), and resilience in a sample of help-seeking individuals in Puerto Rico. METHODS/STUDY POPULATION: This is an analytic, cross sectional design. Adults receiving health services will participate

the study. Recruited participants will provide informed consent during a visit to a community mental health clinic or community hospital. They will complete a demographic document and four retrospective questionnaires about the variables of study. RESULTS/ ANTICIPATED RESULTS: We expect that a high rate of potentially traumatic events (PTEs) is associated with an increased rate of posttraumatic stress symptoms (PTSS). We also expect that a high rate of PTSS is associated with an increased rate of posttraumatic growth low rates of PTSS and PTG. DISCUSSION/SIGNIFICANCE OF IMPACT: This is a first step in the development of effective, clearly targeted interventions, specifically designed to treat negative effects, and also to facilitate positive change and resilience after PTE exposure.

3049

Prenatal care as a protective factor for preterm birth and smoking during pregnancy in nulliparous patients: a propensity score analysis

Alexandra Noel Houston-Ludlam ${ }^{1}$, Alison G. Cahill, Kathleen K. Bucholz and Andrew C. Heath

${ }^{1}$ Washington University in St. Louis, Institute Of Clinical and Translational Sciences

OBJECTIVES/SPECIFIC AIMS: Preterm birth rates have been rising in the United States, and reducing preterm birth is a high-priority clinical and public health concern. There are no existing strategies to reduce preterm birth in nulliparous individuals. The present study aims to evaluate prenatal care as a protective factor for preterm birth in this population. METHODS/STUDY POPULATION: Missouri birth record data for child birth years 1993-2016 were used to create a sample of 325,088 singleton births to nulliparous women, themselves born in MO 1975-1985. Logistic regressions, stratified by maternal race (White, African-American, Asian, American Indian/ Alaskan Native, Other), were used to predict preterm birth $(<37$ weeks gestational age) as a function of 1) initiation of prenatal care of by end of first trimester and 2) Adequacy of Prenatal Care Utilization Index, with sociodemographic covariates of child birth year, maternal age, highest educational level, and marital status (four level variable, including married yes/no, and partner named on birth record, yes/no). Subsequent analyses will use this logistic regression to create a propensity score predicting smoking during pregnancy using birth record parental sociodemographic characteristics, stratified by maternal race. Primary analyses will focus on the role of prenatal care in predicting smoking during pregnancy and preterm birth risk within propensity score stratum. Secondary analyses will consider the role of other risk factors, including maternal pre-pregnancy BMI and maternal DUI history, on preterm birth risk. RESULTS/ ANTICIPATED RESULTS: Preliminary logistic regressions predicting preterm birth were analyzed, stratified by maternal race. In White mothers, preterm birth prevalence was $8.2 \%$, and risk was significantly increased by maternal age $\leq 15$ and $\geq 31$, being unmarried, and by receiving no prenatal care, yet unaffected by timing of prenatal care initiation. For African-American mothers, preterm birth prevalence was $11.9 \%$, and risk was significantly increased by being unmarried and both by not initiating prenatal care by end of first trimester and receiving no prenatal care. Preliminary samples were too small for solid inferences for other races. Anticipated results are that after propensity score match, earlier initiation of prenatal care will show modest protective effect on preterm birth, but other characteristics such as maternal cigarette smoking during pregnancy and DUI status will show stronger effects on predicting preterm birth risk. DISCUSSION/SIGNIFICANCE OF IMPACT: By evaluating the role of prenatal care initiation and delivery on preterm birth, this work provides an evidence base for prenatal care schedules and for understanding the interplay of sociodemographics, healthcare delivery, and individual characteristics in the context of preterm birth risk and potentially reduce negative health outcomes. 